\title{
Inventaire sommaire des sources manuscrites, conservées au Saguenay-Lac Saint-Jean
}

\section{André Côté}

Volume 40, 1973

URI : https://id.erudit.org/iderudit/1007245ar

DOI : https://doi.org/10.7202/1007245ar

Aller au sommaire du numéro

Éditeur(s)

Les Éditions Historia Ecclesiæ Catholicæ Canadensis Inc.

ISSN

0318-6172 (imprimé)

1927-7067 (numérique)

Découvrir la revue

Citer cet article

Côté, A. (1973). Inventaire sommaire des sources manuscrites, conservées au Saguenay-Lac Saint-Jean. Sessions d'étude - Société canadienne d'histoire de l'Église catholique, 40, 9-24. https://doi.org/10.7202/1007245ar

Tous droits réservés @ Les Éditions Historia Ecclesiæ Catholicæ Canadensis Inc., 1974
Ce document est protégé par la loi sur le droit d'auteur. L'utilisation des services d'Érudit (y compris la reproduction) est assujettie à sa politique d'utilisation que vous pouvez consulter en ligne.

https://apropos.erudit.org/fr/usagers/politique-dutilisation/ 


\section{Inventaire sommaire des sources manuscrites, conservées au Saguenay-Lac Saint-Jean*}

Les dépôts d'archives de la province de Québec sont fort nombreux et la plupart contiennent une riche documentation qui attire de plus en plus les spécialistes de diverses disciplines. Cependant, très peu d'entre eux peuvent offrir à leurs visiteurs de marque les instruments de recherches essentiels, tels guides, répertoires ou inventaires ${ }^{1}$.

Les archivistes, généralement en nombre trop restreint, veulent bien satisfaire leurs hôtes, mais ils ne doivent pas oublier leurs fonctions essentielles consistant en pratique à rechercher l'espace vital qui s'amenuise de jour en jour, et à classer même sommairement les nombreuses séries qui encombrent leurs rayons de fortune. Ainsi, les inventaires tant souhaités tardent-ils à venir. Les recherches historiques en subissent le contrecoup, et les orientations nouvelles de l'historiographie qu'on retrouve à l'étranger, notamment en France et aux États-Unis, se taillent difficilement une place dans un tel contexte.

C'est pour répondre à ce double besoin, ressenti à la fois par les chercheurs et les archivistes, que nous avons entrepris de faire l'inventaire sommaire des sources manuscrites, conservées dans notre région du Saguenay-Lac-Saint-Jean. Cet inventaire, en cours depuis deux années, a déjà donné quelques résultats positifs, et c'est pour cette raison que nous avons jugé bon d'en faire l'objet de l'exposé qui va suivre. Nous nous proposons de vous donner d'abord le plan général de notre travail, pour vous parler en second lieu des méthodes que nous avons mises au point; finalement, nous vous ferons part, à titre d'exemple, de l'inventaire des archives paroissiales et des orientations de recherche que nous pouvons déceler dès maintenant.

* Cet inventaire a débuté en jan vier 1972; l'Université du Québec à Chicoutimi nous a accordé, à ce moment-là, une subvention qui nous a permis de faire les premiers pas. Depuis le mois d'avril 1973, nous effectuons notre triavail grâce à une subvention importante qui nous vient du Conseil des Arts du Canada (Ottawa).

1 Les guides, répertoires ou inventaires de dépôts, qui ont fait l'objet d'une publication, sont mentionnés dans: André Beaulieu, Jean Hamelin et Benoît Bernièr, Guide d'Histoire du Canada, Québec, Presses de l'Université Laval, 1969 ("Cahiers de l'Institut d'Histoire, n ${ }^{\circ} 13$ "), 540 p., p. 189.213; François BEAUdin, "Sources manuscrites relatives à l'histoire du Canada ", Archives, 70.1 (1970), p. 46-58; nous tenons à ajouter gue la revue Archives publie couramment des inventaires de tonds qui ne doivent pas être ignorés des chercheurs. 


\section{I - DESCRIPTION GÉNÉRALE DE L'INVENTAIRE}

L'inventaire sommaire que nous menons concerne l'ensemble des documents publics et privés, conservés au Saguenay-Lac-Saint-Jean. Nous nous intéressons aux institutions, aux administrations diverses et, de façon générale, à toute maison qui conserve des documents d'ordre historique. Les archives religieuses nous préoccupent donc au même titre que les archives profanes.

Le territoire sur lequel porte cet inventaire couvre les limites actuelles du diocèse de Chicoutimi, ce qui exclut, bien entendu, la Côte-Nord et le comté de Charlevoix ${ }^{2}$. Cette division, à connotation religieuse, s'avérait impérative, étant donné l'évolution des structures administratives de la région. En effet, l'histoire juridique des institutions québécoises nous enseigne que l'autorité civile, depuis les débuts de la colonisation française, a suivi et non précédé l'autorité religieuse; de plus, les cadres administratifs de cette même autorité civile ont généralement épousé les cadres administratifs de l'autorité religieuse ${ }^{3}$. Il n'est donc pas étonnant que nous tenions compte de cette réalité dans l'inventaire des documents manuscrits qui ont eux-mêmes été produits par ces administrations.

Les documents religieux, qui font l'objet de notre inventaire, se retrouvent principalement dans les presbytères, les communautés et les

2 Le diocèse de Chicoutimi a été érigé le 28 mai 1878 par le pape Léon XIII. Il est une division du territoire de l'archidiocèse de Québec et fait partie de la Province ecclésiastique de Québec depuis son érection. A l'origine il groupait les comtés de Chicoutimi, de Charlevoix et la partie du comté de Saguenay située à l'ouest de la rivière Portneuf. La Bulle d'érection du nouveau diocèse du Golfe Saint-Laurent (aujourd'hui le diocèse de Hauterive), en date du 24 novembre 1945, détachait du diocèse les six paroisses de la Côte-Nord. Par un décret, en date du 23 juin 1951, le comté de Charlevoix est détaché du diocèse de Chicoutimi et réuni à l'archidiocèse de Québec. Le 9 janvier 1953, les limites communes des diocèses d'Amos et de Chicoutimi sont mieux définies : l'expression "Hauteurs des terres" doit désormais s'entendre des limites qui séparent les comtés Abitibi-Est et Abitibi du comté Roberval. Le territoire du diocèse a une superficie de 35,000 milles carrés et il comprend, au niveau provincial, les circonscriptions électorales suivantes: Chicoutimi, Dubuc, JonquièreKénogami, Lac Saint-Jean et Roberval. Sa population, en novembre 1972, était de 256,962 (cf. Diocèse de Chicoutimi, Annuaire diocésain, 1972-1973, Chicoutimi, Êvêché de Chicoutimi, 1973 , p. 5-8). L'histoire de cette région remonte au début du XVII ${ }^{\mathrm{e}}$ siècle; durant tout le régime français, les activités des Blancs se limitent au commerce des fourrures et à l'évangélisation des Montagnais. Après la Conquête anglo-américaine (1760), on continua à se préoccuper des fourrures; cependant, à compter des années 1837-1840, des colons de Charlevoix s'établirent à $\mathrm{La}$ Grande-Baie, et ce fut le début du grand mouvement de colonisation qui ne s'est arrêté qu'au premier quart du $\mathrm{XX}^{\mathrm{e}}$ siècle. L'occupation du territoire est donc relativement récente (elle date de 136 ans); avec elle, coïncident les débuts des structures ad. ministratives régionales, sur le plan religieux et profane, et aussi les débuts des séries de documents qui y sont conservés.

3 Il existe sur cette question de nombreux ouvrages d'inégale valeur; on recommande plus particulièrement: Edmond LAREAU, Histoire du droit canadien, depuis les origines de la colonie jusqu'à nos jours, Montréal, Périard, 1888-1889, 2 vol., I: Domination française; II : Domination anglaise; Structures sociales du Canada français, Etudes de membres de la section I de la Société royale du Canada, Guy Sylvestre éd., Québec, Presses de l'Université Laval, 1966, $120 \mathrm{p}$. 
maisons d'enseignement; les papiers d'hommes d'Église, qui peuvent être retrouvés, sont également inventoriés. Les documents profanes, d'ordre public ou privé, se retrouvent dans les services administratifs des gouvernements locaux, provincial et fédéral; ils se retrouvent aussi dans les entreprises industrielles, les hôpitaux et les familles. Les papiers des associations qui nous sont accessibles sont également inventoriés.

Les archives des presbytères - dites archives paroissiales - sont celles qui nous occupent surtout depuis le début de notre inventaire. N'en parlons pas davantage pour le moment, car nous aurons l'occasion de revenir sur ce propos, au cours de notre exposé.

Les archives des communautés religieuses ${ }^{4}$ seront abordées d'après le plan que nous avons mis au point lors d'une récente étude, faite sur l'établissement des trappistes dans la province de Québer ${ }^{5}$. Le schéma qu'on présente dans cette analyse divise les documents des trappistes en trois parties: en premier lieu, viennent les archives socio-religieuses; en second lieu, les archives économiques, et finalement, les documents qui se rapportent à l'environnement du monastère. Nous savons par ailleurs qu'un tel modèle ne pourra regrouper sans distinction les archives de toutes les communautés, car plusieurs ont des fonctions bien différentes de celles des trappistes.

Les maisons d'enseignement qui retiennent notre attention sont les écoles normales ${ }^{6}$, les instituts familiaux ${ }^{7}$, les écoles de Génie et de commerce de Chicoutimi. Nous avons terminé l'inventaire des deux dernières institutions qui ont dispensé un enseignement universitaire au Saguenay durant une vingtaine d'années, avant l'ouverture de l'Université du Québec à Chicoutimi. Cet inventaire permettra à l'un de ses professeurs de

4 Les communautés, établies dans le diocèse, se répartissent comme suit: communautés cléricalés, 18; communautés de Frères, 7; communautés de Femmes, 17; instituts séculiers, 2. Lorsqu'une communauté possède plusieurs maisons, l'une d'entre elles - appelée maison locale ou provinciale - dirige généralement l'ensemble des autres établissements régionaux. Il arrive aussi que la maison mère d'une communauté soit située dans le diocèse; c'est notamment le cas pour l'Institut des Sœurs de Notre-Dame du BonConseil, et pour la Congrégation des Sœurs de Saint-Antoine-de-Padoue. Il est évident que nous ne pourrons inventorier les archives de toutes les maisons religieuses du diocèse; nous visiterons uniquement les maisons locales et provinciales de même que les maisons mères. La liste des communautés religieuses est publiée dans : Diocèse de Chicoutimi, Annuaire diocésain, 1972-1973, p. 81-109.

5 André Côté, L'ordre de Cîteaux et son établissement dans la province de Québec, depuis la Révolution française jusqu'à 1935, thèse de maîtrise soutenue à l'Université Laval en février 1972. lviii - 204 p.. cartes, plans, photos, h.-t.

6 Ces écoles, aujourd'hui disparues, furent au nombre de six; elles étaient situées dans les villes de Chicoutimi (on y retrouvait deux maisons), Arvida, Alma, Roberval et Dolbeau.

7 Ces écoles sont également disparues depuis quelques années. Elles étaient au nombre de deux et elles se trouvaient toutes deux à Chicoutimi. 
publier sous peu l'Histoire du mouvement universitaire au Saguenay ${ }^{8}$. Les archives du Séminaire de Chicoutimi, cette grande maison que nous avons fréquentée avec statut de pensionnaire durant huit années et dont nous gardons le meilleur des souvenirs, ne feront pas partie de notre inventaire, du moins pour le moment, car des équipes d'étudiants, bien dirigées, s'adonnent actuellement à ce travail. Les documents, conservés par les maisons d'enseignement, sont regroupés sous les vedettes suivantes: direction générale, biens immobiliers, services financiers et comptabilité, services commerciaux, personnel, et clientèle étudiante.

Les papiers des hommes d'Église seront inventoriés de la même façon que les papiers de familles. Ils seront groupés d'après ces vedettes: biens immobiliers, comptabilité, correspondance générale, et documents personnels.

Si les documents religieux occupent une place importante dans notre inventaire, nous ne délaissons pas pour autant les documents profanes. Ceux qui font d'abord l'objet de notre préoccupation sont accumulés par les services administratifs des gouvernements locaux. C'est ainsi que nous sommes amenés à inventorier les archives des municipalités et celles des commissions scolaires.

Les archives des municipalités sont regroupées sous 13 vedettes générales qui s'énoncent comme suit: règlements généraux, procèsverbaux du conseil ou registres de délibérations, pièces justificatives ac'compagnant les procès-verbaux, rôles d'évaluation et rôles d'imposition foncière, comptes de taxe, actes notariés, permis de construction et plans de construction approuvés, livres de comptes (i.e. états financiers), chèques, factures et commandes, dossiers des employés, plans cadastraux, rapports $d^{\prime}$ 'incendie, et listes de paie ${ }^{9}$.

Les archives des commissions scolaires forment, quant à elles, les vedettes suivantes: direction générale, registre des assemblées, rapports des commissaires d'écoles, comptes publics, personnel enseignant, et clientèle étudiante.

Les services administratifs des gouvernements provincial et fédéral contiennent des archives historiques fort intéressantes, comme le démontrent les premières visites qui y ont été effectuées. Nous n'avons pas encore établi de plan de travail précis sur ce point mais nous désirons le faire a vec la collaboration du conservateur des Archives nationales du Québec.

8 Cette publication sera faite par un de nos collègues (Jean-Guy Genest), grâce à une subvention qui lui fut accordée par la fondation de l'Université du Québec à Chicoutimi.

$9 \mathbf{M}^{\text {me }}$ Murielle Doyle-Frenière, archiviste de l'hôtel de ville de Québec, a bien voulu nous aider à établir ces vedettes. Nous lui en sommes fort reconnaissant. 
Les archives d'associations profanes ${ }^{10}$ sont regroupées sous les vedettes qui suivent: fondation, composition (i.e. membres), administration, comptabilité, correspondance expédiée, correspondance reçue, et dissolution de l'association.

Les papiers des entreprises industrielles ${ }^{11}$ peuvent être groupés sous 13 vedettes, soit: constitution de l'affaire, conseil, direction générale, domaine, matériel d'exploitation, approvisionnements et fabrications, service financier, comptabilité, correspondance, contentieux, services commerciaux, personnel, et finalement, services d'études.

Le dernier volet de notre inventaire concerne les archives hospitalières ${ }^{12}$. Cette catégorie de documents est présentée d'après les vedettes suivantes: réglementation générale et locale, personnel laïc de toutes catégories, personnel religieux hospitalier, corps médical hospitalier, enseignement hospitalier, administration générale, comptabilité, biens immobiliers, population (i.e. malades hospitalisés), et vient, en dernier lieu, l'aumônerie.

Les dépôts d'archives régionales, qu'ils soient d'ordre public ou privé, se présentent sous des formes diverses, et leur seule caractéristique commune - il ne faut pas s'en étonner - demeure l'abense de classement rationnel. Aussi, leur inventaire, même sommaire, s'avérerait-il non

10 Voici les principales associations dont nous nous proposons d'inventorier les

- Association des constructeurs Saguenay-Lac Saint-Jean

- Association des enseignants du Saguenay

- Association des enseignants du Lac Saint-Jean

- Association Lacordaire du Canada

- Cercle régional des jeunes agriculteurs

- Chevaliers de Colomb

- Clinique Domrémy

- Comité paritaire de l'industrie de la construction

- Fédération des chantiers coopératifs du Saguenay

- Société diocésaine de colonisation

- Société nationale du Saguenay-Lac Saint-Jean (auparavant : La société SaintJean-Baptiste)

- Société Saint-Vincent-de-Paul

- Syndicats nationaux (C.S.N.)

- Union catholique des cultivateurs (U.C.C.)

11 Voici les principales entreprises dont nous allons inventorier les papiers:

- Aluminium Company of Canada (Arvida, Isle Maligne)

- Caisses populaires Desjardins

- Chaîne Coopérative du Saguenay (Saint-Bruno)

- La Compagnie Price Limitée (Kénogami, River bend)

- Consolidated Bathurst Limited (Port-Alfred)

- Domptar Newsprint Limited (Dolbeau)

- St. Raymond Paper Limited (Desbiens)

12 Les hôpitaux de la région sont situés dans les villes de Chicoutimi (1884: date de fondation de l'hôpital), Roberval (1918), Arvida (1927), Alma (1954), Jonquière (1955), Dolbeau (1955) et Bagotville (1970). 
réaliste - voire impossible - s'il n'était effectué d'après une méthode rigoureuse qui est mise au point pour chaque catégorie de fonds, ayant un même contenu.

\section{II - MÉTHODE DE TRAVAIL}

La mise au point d'une méthode de travail relative à chaque catégorie de fonds, ayant un même contenu, comprend trois phases:

$\mathrm{l}^{\text {re }}$ travail préliminaire;

$2^{\mathrm{e}} \quad$ rédaction d'un questionnaire provisoire;

$3^{e} \quad$ rédaction d'un questionnaire définitif.

Durant le travail préliminaire, on consulte d'abord des ouvrages pouvant expliquer l'évolution des structures d'une administration donnée, pour lire ensuite des écrits qui traitent des archives qui peuvent y être retrouvées.

Ainsi, avant d'aborder les archives paroissiales, avons-nous consulté l'Histoire du droit canadien d'Edmond Lareau ${ }^{13}$; le code de Droit paroissial et fabricien de Migneault et de Pouliot ${ }^{14}$; le Code municipal de Bédard ${ }^{15}$; l'Appendice au rituel romain ${ }^{16}$, les Mandements des évêques du diocèse ${ }^{17}$, les Décrets du premier synode de Chicoutimi ${ }^{18}$, les Annuaires du diocèse de Chicoutimi $^{19}$, la série complète du Canada ecclésiastique ${ }^{20}$, le Dictionnaire historique et géographique des paroisses, missions et municipalités de la province de Québec ${ }^{21}$, et finalement, le monumental ouvrage de $\mathrm{M}^{\mathrm{gr}}$ André

13 Montréal, Périard, 1888-1889, 2 vol.

14 P.-B. Migneault, Le droit paroissial, étant une étude historique et légale de la paroisse catholique, de sa création, de son gouvernement et de ses biens (...), Montréal, Beauchemin, 1893, 690 p.; Jean-François Pouliot, Traité de droit fabricien et paroissial, Étude critique de législation comparée, Montréal, Wilson et Lafleur, 1936, $1010 \mathrm{p}$. $1898,663 \mathrm{p}$.

15 J.-E. BÉdard, Code municipal de la province de Québec (...), Montréal, Théoret,

16 Appendice au rituel romain, réédité par ordre des Pères du premier concile plénier de Québec, Québec, Imprimerie de L'Action sociale limitée, 1919, 278 p. Les quatre éditions de l'Appendice au rituel romain, parues respectivement en 1853, 1874, 1890 et 1919, renferment les matières de la deuxième partie et le chapitre II de la troisième partie, Des Formules, du rituel de Saint-Vallier, deuxième évêque de Québec.

17 Mandements, lettres pastorales et circulaires des évêques de Chicoutimi (de 1878 à nos jours), 22 vol.

18 Publiés et promulgués par son Excellence révérendissime Monseigneur Georges Melançon, évêque de Chicoutimi, Chicoutimi, Chancellerie de l'évêché, 1954, 439 p.; nous y retrouvons 400 décrets et 30 appendices.

19 Ces annuaires diocésains ont commencé à paraître en 1915; leur forme a varié à quelques reprises au cours des décennies; celle que nous connaissons présentement remonte à 1963.

20 Ces répertoires sont publiés par la maison Beauchemin de Montréal, depuis 1887.

21 Écrit par Hormidas Magnan, Arthabaska, Imprimerie d'Arthabaska, 1925, 735 p. 
Simard, intitulé: Les évêques et les prêtres séculiers au diocèse de Chicoutimi, 1878-1968 ${ }^{22}$.

En plus de nous relater l'histoire, trop souvent méconnue, des institutions juridiques, politiques et religieuses de la province de Québec, ces ouvrages nous expliquent avec maints détails la lente évolution des structures paroissiales qui existent depuis le milieu du XVII e siècle. Parmi les autres renseignements essentiels que nous pouvons encore $y$ relever, signalons la liste des paroisses actuelles ou disparues, la liste des prêtres séculiers ou réguliers qui ont exercé des fonctions curiales, les droits et les devoirs des paroissiens vis-à-vis leurs curés, et vice-versa. Nous avons même retrouvé les diverses catégories de documents qui doivent être conservées dans les presbytères du diocèse de Chicoutimi ${ }^{23}$.

Avec de telles précisions, il fut possible d'établir sur des bases solides un questionnaire provisoire d'inventaire. Ce dernier fut mis à l'essai dans quatre paroisses; deux d'entre elles étaient centenaires: il s'agit de SaintFrançois-Xavier de Chicoutimi (1845) et de Saint-Dominique (1866); les deux autres, étant des fondations récentes: il s'agit de Sainte-Thérèse (1927) et de Saint-Mathias d'Arvida (1955). Après avoir analysé les diverses catégories de documents qu'on y retrouvait, on en est arrivé à les regrouper en trois grandes sections, soit: Archives de la paroisse, Archives de la Fabrique, et Archives du personnel religieux.

À l'intérieur de chaque section, on dispose les catégories de documents - qui deviendront probablement des séries lorsque le classement sera effectué - d'après la structure juridique et administrative de la paroisse. On voit encore ici l'importance des ouvrages déjà mentionnés. Pour chaque catégorie de documents, on indique :

$1^{\text {re }}$ le nom de la catégorie qui peut concerner un ensemble de dossiers ayant le même contenu ou bien des registres semblables, tels les registres d'état civil ou de confirmations;

$2^{\mathbf{e}}$ les dates extrêmes avec mention de l'année et du jour;

$3^{\text {e }}$ le nombre de volumes ou de chemises;

$4^{\mathrm{e}}$ le nombre de pouces ou de pieds linéaires;

$5^{\text {e }}$ le lieu de conservation.

Comme on peut le constater, les informations qu'on donne au chercheur demeurent sommaires; cependant, ce dernier en aura suffisamment pour entrevoir de sérieuses analyses. 
L'inventaire des autres fonds d'archives est effectué suivant un procédé semblable qu'il serait trop long de développer dans le cadre de l'exposé que nous nous sommes fixé. Signalons tout de même l'utilisation du Manuel d'archivistique ${ }^{24}$ qui s'est a vérée nécessaire lors de l'élaboration de certains questionnaires provisoires.

La méthode de travail que nous avons mise au point - et que nous venons d'appliquer aux archives paroissiales, à titre d'exemple - paraît fort rigoureuse, mais elle semble requise à toute présentation uniforme de documents non encore classés.

\section{III - ARCHIVES PAROISSIALES ET ORIENTATIONS DE RECHERCHE}

Les archives, conservées dans les presbytères, sont inventoriées som. mairement à l'aide d'un questionnaire comprenant 35 pages. La première page donne l'adresse et le numéro de téléphone du presbytère, et fournit la liste complète des prêtres séculiers ou réguliers qui ont exercé des fonctions curiales, depuis les débuts de la paroisse jusqu'en 1973.

Dès la deuxième page, arrive la première section de l'inventaire, dite: Archives de la paroisse. Cette section renferme 21 vedettes-matières, dont chacune correspond à des dossiers, des registres ou des cahiers de même contenu.

Les quatre premières vedettes regroupent les décrets relatifs à la fondation ou à l'organisation générale de la paroisse; ainsi retrouvons-nous les documents qui concernent l'érection canonique ou civile, la bénédiction d'une église, d'une chapelle, d'une école, d'un cimetière, d'un chemin de croix, etc.

Les vedettes, dont les numéros vont de cinq à neuf, portent les titres suivants : "authenticité des reliques", "consécration de l'église", " conversions au catnolicisme (ou abjurations)", "grandes retraites paroissiales", et " ordinations".

Dans la vedette, intitulée: "authenticité des reliques", nous avons noté les reliques conservées dans chaque paroisse, de même que les dates d'acquisition de chacune d'elles. Une analyse, faite dans cette direction, permettrait de connaître les diverses dévotions qui furent pratiquées dans nos paroisses saguenayennes. On pourrait peut-être y déceler des

24 Manuel d'archivistique. Théorie et pratique des archives publiques en France, ouvrage élaboré par l'Association des archivistes français, ministère des Áffaires culturelles, Direction des archives de France, Paris, S.E.V.P.E.N., 1970, 805 p. 
dévotions propres à chaque groupe social, que ce soit dans le monde rural ou dans le monde urbain. Quoi qu'il en soit, toute recherche sérieuse projetterait certes quelque clarté sur la sensibilité religieuse.

La vedette intitulée: "consécration de l'église" contient des documents dont le contenu est défini par le titre lui-même. On doit signaler que seules six paroisses sur 95 ont vu leur église consacrée jusqu'à maintenant. Trois conditions sont requises pour qu'une paroisse puisse bénéficier de cet honneur. Il faut :

$1^{\mathbf{e}}$ que l'église soit payée en entier;

$2^{\mathbf{e}}$ qu'elle soit à l'épreuve du feu;

$3^{\text {e }}$ que l'autel soit érigé sur des fondations qui touchent directement au sol.

Si l'une des conditions fait défaut, l'èglise paroissiale n'est pas consacrée.

Les dossiers concernant les "conversions au catholicisme (ou abjurations)" sont secrets. Aussi, sont-ils conservés sous clef dans le bureau du curé; nous devons généralement nous contenter de les voir sans consulter aucune pièce. Les "retraites paroissiales" et les "ordinations" sont généralement mentionnées dans les livres de prônes; les registres ou dossiers qui peuvent être constitués à leur sujet demeurent très rares.

Les autres vedettes, portant les numéros dix à quinze, sont intitulées : "baptêmes, mariages et sépultures", "investigations prénuptiales", "confirmations", "annonces et prônes ", " bancs ", et "bans de mariage".

Les registres des baptêmes, mariages et sépultures, constituent, d'après Pierre Goubert, la plus massive des sources de l'histoire des "peuples ${ }^{25}$ ". La fréquente précision des actes de mariage nous donne l'âge des conjoints et leur origine démographique. Lorsque les actes de sépulture ne se réduisent pas à une sèche mention, des statistiques d'âge au décès ou de situation matrimoniale peuvent être envisagées. Les actes de baptêmes permettent l'étude des naissances illégitimes, des naissances gémellaires et du taux de masculinité à la naissance. L'ensemble des actes fournit le moyen de recouper, de fortifier les enquêtes de structure sociale; il peut fournir d'abondants matériaux à des études de linguistique historique, et, par l'intermédiaire des noms de baptême, il permet l'analyse de la piété populaire ${ }^{26}$.

${ }_{25}$ Cf. Cent mille provinciaux au XVII siècle, Beauvais et le Beauvaisis, de 1600 à 1730, (Paris), Flammarion, (1968), (Coll. "Science de l'Histoire"), 439 p., p. 50.

26 Ibid., p. 5l. Les autres études de Goubert qui nous éclairent sur ce point sont les suivantes: L'Ancien Régime, Tome I: la société, Paris, Colin, (1969), (Coll. " Un), 2e éd., p. 35 52; "Le poids du monde rural ", dans : Histoire économique et sociale de la France, Tome II: Des derniers temps de l'âge seigneurial aux préludes de l'âge industriel (1660-1789), par Ernest Labrousse, Pierre LÉon, et alii, Paris, P.U.F., (1970), p. 3-80. 
Les "dossiers d'investigations prénuptiales" ont été constitués à compter de l'année 1943 afin de mieux contrôler les soldats qui désiraient contracter mariage. Chaque dossier est formé d'un nombre de pièces bien déterminées; il en comprend quatre lorsque les fiancés sont majeurs, et cinq, si ces derniers sont encore mineurs. La première pièce, formée de quatre pages, s'intitule: enquête prénuptiale; elle est divisée en deux parties: l'une s'adresse à la femme, et l'autre, à l'homme. Cette enquête, suivant les mots de la dite formule, "a pour but d'établir et de consigner les données d'un projet de mariage: l'identité des personnes, leur liberté de contracter mariage, leur préparation au mariage chrétien, leurs intentions quant aux éléments essentiels de l'engagement matrimonial ". La deuxième pièce, formée de deux pages, donne l'identité des futurs conjoints et signale leur capacité juridique, leur liberté d'état, et leur liberté de consentement; elle mentionne également les publications et les dispenses de bans, l'arbre généalogique et le Nihil obstat concernant la célébration du mariage.

Le consentement au mariage des mineurs, accordé par les parents, le tuteur ou le curateur, l'avis de publication de mariage et l'attestation de la célébration forment les dernières pièces du dossier.

Ces enquêtes prénuptiales, faites depuis déjà 30 ans, ont constitué une masse de documents qui couvrent parfois 5 pieds de rayonnage. Leur classement, effectué généralement d'après l'ordre alphabétique du futur conjoint, permettra au chercheur de reconstituer facilement les familles pour entreprendre ensuite diverses études socio-religieuses.

Les "registres de confirmations" contiennent les noms des confirmés, et précisent, pour chaque enfant, le nom de la mère, celui du père, du parrain et de la marraine. S'ils sont bien analysés et intégrés dans l'ensemble de la documentation, ils permettront de cerner l'évolution religieuse de chaque membre de la famille. Ces registres prennent d'autant plus d'importance qu'ils sont souvent les seuls à mentionner l'observance rigoureuse d'un sacrement, durant cette longue période qui s'écoule depuis le baptême jusqu'au mariage.

Les "prônes" des dimanches et des jours de fêtes, entendus par tous les paroissiens, jusqu'à ces dernières années tout au moins, sont consignés dans des cahiers de petit format - d'où leur nom de livres de prônes jusqu'aux années 1960; ils sont imprimés, depuis lors, sur des feuillets qu'on distribue à la porte de l'église. Les curés, en plus de bien conserver leurs feuillets paroissiaux, prennent soin de les regrouper, puis de les relier, à la fin de chaque année.

Il va sans dire que les prônes fournissent une intéressante documentation qui touche aux préoccupations du clergé face à la vie quotidienne 
des paroissiens. Aussi, est-il recommandé aux pasteurs "de les enregistrer dans des cahiers solidement reliés, de se servir pour les y inscrire d'une bonne encre noire, et non pas d'une encre de couleur, encore moins d'un simple crayon, et d'éviter, sous prétexte de brièveté, une sorte de sténographie qui rend le texte inintelligible ${ }^{27}$ ". Les curés doivent également y indiquer, au moins d'une façon sommaire, les remarques diverses qu'ils jugent bon de faire et les avis spéciaux qu'ils donnent à leurs fidèles.

Une analyse sommaire des livres de prônes, effectuée dans une trentaine de paroisses, a démontré que le curé informait ses paroissiens, non seulement sur les activités religieuses de la semaine, mais aussi sur toute autre activité d'ordre profane qui pouvait les intéresser. Ainsi avons-nous retrouvé les thèmes suivants aux archives paroissiales de Saint-Jérôme: mois du Rosaire, neuvaines, expositions du saint Sacrement, promesses de mariages, convocations d'assemblées, quêtes spéciales, heures d'adoration, paiement des bancs, abonnement au journal L'Action catholique, élection des marguilliers, parties de cartes, services anniversaires, jeûnes et abstinence, mauvais endroits fréquentés, mauvais livres, confessions, soirées dramatiques et musicales, prêt d'honneur pour les étudiants, vêpres du dimanche soir, souper paroissial, intentions de messes, itinéraires et statistiques de visites paroissiales, assistance à la messe le dimanche et en semaine, cours donnés aux adultes, etc.

Les commentaires, écrits sur chacun de ces thèmes, sont d'une brièveté remarquable; cependant, il est permis de croire qu'ils devaient servir uniquement à orienter l'exposé, car bien des prêtres, connaissant l'art de l'éloquence, devaient sans doute parler vivement de certains propos.

Les "livres de bancs", autre vedette de notre questionnaire, indiquent le prix de chaque banc, et précisent le nom du locataire et la date du bail. Les "bans de mariages" sont généralement mentionnés dans les livres de prônes et dans les dossiers des investigations prénuptiales; aussi, sont-ils rarement consignés dans des registres spéciaux.

Les vedettes, portant les numéros 16 à 20 , ont les titres suivants: "dîme et capitation", "intentions de messes", "livre des âmes", "chronique paroissiale", et finalement, "confréries ou associations pieuses".

Les "cahiers de dîme et de capitation" permettent de contrôler, parfois sur une longue période, les donations qui ont été faites, soit en nature soit en argent, au pasteur de la paroisse. Une simple comparaison, établie 
entre les donations et les revenus des paroissiens, que ceux-ci proviennent de leur ferme ou de leur métier, permettra d'établir le pourcentage annuel des donations par rapport à l'ensemble des revenus d'une famille. Il sera sans doute possible de cerner également l'évolution de ce devoir, si souvent négligé, qui s'est implanté avec difficultés à l'époque de $\mathbf{M}^{\mathrm{gr}}$ de Laval.

Les “ intentions de messes " sont généralement mentionnées dans les livres de prônes; il arrive aussi qu'elles soient consignées dans des cahiers spéciaux.

Les “ livres des âmes ", dits aussi «livres de visites paroissiales ", donnent la liste des membres de chaque famille, le nombre de communiants et de non-communiants, le montant de la dîme et de la capitation si des "cahiers de dîme" ne sont pas tenus, et finalement, le montant de l'offrande, faite à l'Enfant-Jésus. La mise à jour de ces livres devait sans doute s'avérer difficile puisqu'un système de fiches a graduellement remplacé ces derniers, au début des années 1960. La nouvelle formule donne beaucoup plus d'informations sur les familles que l'ancienne; cependant, la mise à jour des fiches, effectuée lors des visites paroissiales, amène la disparition de nombreux renseignements, consignés l'année précédente. Il va sans dire qu'une telle pratique risque fort de gêner le travail des chercheurs.

Les "chroniques paroissiales", rédigées de la main même du prêtre, sont plutôt rares; celles qui ont été retrouvées, notamment aux archives de Sainte-Anne (Chicoutimi-nord), de Saint-Cœur de Marie et de SaintGédéon, demeurent pleines d'intérêt.

Les papiers des "confréries" ou des "associations pieuses " qui ne sont pas encore éteintes appartiennent généralement au président desdites confréries ou associations. Aussi, leur consultation devient-elle moins facile. Les confréries ou les associations qui cessent toute activité voient à l'occasion leurs papiers déposés au presbytère. Dans une telle circonstance, ces documents nous intéressent au même titre que les autres manuscrits.

La dernière vedette de cette section est intitulée: "autres documents"; elle regroupe les papiers d'ordre religieux qui sont propres à chaque paroisse et qui ne trouvent guère leur place ailleurs.

La seconde section du questionnaire s'intitule: "Archives de la Fabrique"; on y retrouve les documents financiers, les papiers qui touchent aux cimetières, les divers plans, et finalement les œuvres d'art.

Les documents d'ordre financier comprennent les registres de délibération de la Fabrique et les registres de comptabilité. Les premiers contiennent non seulement les résolutions des marguilliers mais encore 
des sujets fort variés, tels correspondance, jugement de cour, requêtes diverses, etc. Les seconds font part des recettes et des dépenses annuelles de la Fabrique; ils sont généralement bien conservés et remontent souvent aux premières années des paroisses.

Les actes notariés qui attestent la propriété foncière de la Fabrique se retrouvent rarement aux presbytères. Cependant, les chercheurs n'en sont pas déroutés pour autant, puisqu'ils peuvent les retracer au bureau d'enregistrement du district judiciaire où se trouvent situées lesdites paroisses.

Les documents écrits, se rattachant aux cimetières, existent dans certains presbytères. Les archives de Saint-Jérôme conservent, entre autres, un registre qui donne la liste des corps exhumés du vieux cimetière et inhumés dans le nouveau, à compter des années 1918 jusqu'à 1949; il précise également l'ensemble des lots qui ont été concédés aux paroissiens durant la même période. Aux archives de Saint-Bruno, on a retrouvé les règlements des cimetières paroissiaux qui portent les titres suivants: respect et bon ordre à observer dans le cimetière, droit d'inhumation, lots et fosses, terrain réservé, et dispositions finales. Le presbytère de la paroisse Saint-Sacrement (Alma) conserve les permis d'inhumation et de transport de cadavres, à compter du 5 mars 1949 jusqu'au 20 octobre 1959.

De semblables documents, ajoutés aux fameux sermons sur la mort et l'enfer, qui étaient prononcés par des prédicateurs itinérants lors des grandes retraites paroissiales ou à l'occasion des mercredis des cendres, et qui effrayaient tant nos jeunes oreilles attentives, devraient éclairer, croyons-nous, la vision de la mort et de l'au-delà qui était certes présente dans chaque foyer. Des études sérieuses montreront peut-être que cette vision se rapproche de celle qui avait cours aux époques médiévales et modernes ${ }^{28}$.

Les autres vedettes de cette section regroupent les plans et les œuvres d'art religieux. Les plans touchent le plus souvent aux édifices de la Fabrique; il arrive parfois qu'ils se rapportent à d'autres édifices de la paroisse et aux édifices de Fabrique d'une autre paroisse, comme le cas s'est notamment présenté aux archives de Saint-François-Xavier de - Chicoutimi. L'inscription des plans sur notre questionnaire est effectuée de cette façon: on indique d'abord leur titre et la date de leur confection,

28 Sur cette question, on pourra consulter: J. Huizinga, Le déclin du Moyen Age, traduit du hollandais par J. Bastin, Paris, Payot, 1961 ("Bibliothèque historique "), 406 p.; Robert Mandrou, De la culture populaire aux XVII et XVIII siècles, La Bibliothèque bleue de Troyes, (Paris), Stock, (1964), 223 p.; La France aux XVII et XVIII siècles, Paris, P.U.F., 1967 (Coll. "Nouvelle Clio ", n 33), 335 p. 
pour signaler ensuite le nom de leur auteur, ce dernier étant généralement un architecte ou un ingénieur.

Dans le vaste domaine des œuvres d'art, on se préoccupe surtout du mobilier des presbytères et des églises, des vases sacrés, des tableaux et des sculptures, tels statues, autels, chemins de croix, etc.

Un intérêt semblable devrait permettre aux spécialistes de cette discipline de connaître et d'expliquer surtout l'évolution de l'art religieux dans cette région. En constatant les formes actuelles - en particulier. l'architecture remarquée des nouveaux temples religieux - et en sachant que l'art est l'expression d'un besoin de l'être humain, à une certaine époque, selon les mots de Lucien Febvre ${ }^{29}$, ces mêmes spécialistes pourront sans doute mesurer l'influence des courants extérieurs sur les tendances locales; leur analyse révélera peut-être l'existence d'un art typiquement saguenayen qui s'est graduellement formé au cours des générations pour influencer ensuite les régions voisines. Un tel phénomène ne serait certes pas nouveau puisqu'il s'est produit plusieurs fois en France, et particulièrement à l'époque de la Renaissance ${ }^{30}$. Si une telle étude s'avère trop difficile dans le contexte actuel, on peut tout au moins espérer que les historiens de l'art se préoccuperont des changements qui surviennent depuis déjà une décennie au niveau de la sensibilité religieuse.

La dernière partie de notre questionnaire, intitulée: "Archives du personnel religieux", regroupe les papiers privés des pasteurs de paroisses. Cé genre de documents se rencontre rarement, étant donné que les prêtres ne sont pas tenus de laisser au presbytère les papiers qu'ils ont accumulés durant leur séjour plus ou moins prolongé. Leur consultation s'avère pourtant essentielle à quiconque désire comprendre les mouvements de colonisation de la province ou la vie quotidienne des paroissiens.

Un seul exemple servira d'illustration à cette idée: lorsque nous avons étudié l'établissement des trappistes de Mistassini, les archives épiscopales de Québec et de Chicoutimi, de même que les sources conservées aux monastères d'Oka et de Mistassini, ont été bien sûr consultées. Lanalyse des documents qui concernaient cette question démontrait, d'une façon évidente, le rôle prépondérant joué par les autorités civiles et religieuses de la province de Québec; cependant, nous n'avions pas l'opinion des quatre prêtres qui ont alors influencé fortement ces mêmes autorités jusqu'à ce que nous ayons retrouvé leurs fonds privés. Il s'agit de

29 Cf. Combats pour l'histoire, Paris, Colin, 1965, (Économies - Sociétés Civilisations), $2^{\mathbf{e}}$ éd., p. 313.

$30 \quad$ Ibid., p. 295-313. 
$M^{\text {sr }}$ Antoine Labelle, commissaire adjoint de l'agriculture et de la colonisation, dont les papiers se trouvent conservés aux Archives nationales du Québec ${ }^{31}$. Vient, en second lieu, M ${ }^{\text {gr }}$ Joseph-Calixte Marquis, gérant de la colonisation au Saguenay et grand ami du curé Labelle, dont les papiers peuvent être consultés aux Archives dü grand séminaire de Nicolet et à celles du monastère de Mistassini ${ }^{32}$. En troisième lieu, se rencontre l'abbé Louis-Joseph Tremblay, curé de Saint-Méthode durant une trentaine d'années, qui voit ses papiers conservés par la Société historique du Saguenay ${ }^{33}$. Finalement, vient le chanoine ThomasGrégoire Rouleau, principal de l'école normale Laval de Québec, qui a laissé ses papiers à la maison où il a œuvré durant 52 ans $^{34}$.

Si la recherche de semblables documents demeure difficile l'heuristique exige en effet beaucoup de temps et de nombreux déboursés - il est par ailleurs souhaitable qu'elle ne soit point délaissée par les historiens. D'un autre côté, les enquêtes les plus soutenues demeureront stériles sans une collaboration efficace des hommes d'Église. Le moment est donc choisi pour inviter les membres du clergé à ne point détruire leurs papiers personnels. S'il arrive que ces documents deviennent trop encombrants, pour des raisons diverses et fort compréhensibles, les dépôts d'archives publiques ou privées pourront les accepter, après une entente faite par-devant notaire. Ce sera désormais avec hésitation que les

31 Le fonds est intitulé: "Labelle, $\mathrm{M}^{\mathrm{gr}}$ Antoine, 1860-1891". Il s'agit d'une correspondance placée dans l'ordre alphabétique et accompagnée d'un index; elle comprend 1482 lettres et forme 14 pouces linéaires; le fonds est contenu dans quatre boîtes. Son analyse donne les renseignements suivants : 1412 lettres ont été reçues de 596 personnes; 70 lettres ont été envoyées par Labelle à 64 personnages; une seule a été envoyée à $\mathbf{M}^{\mathrm{gr}}$ JosephCalixte Marquis: datée du 4 août 1890 et écrite de Paris, elle concerne l'établissement des trappistes au Lac Saint-Jean (boîte $n^{\circ} 2$ ). Cinq lettres ont été écrites par Marquis (boîte $n^{\circ} 3$ ): les trois d'entre elles qui concernent les trappistes du Lac Saint. Jean ont été envoyées à Labelle, alors que ce dernier se trouvait en France; elles sont datées des 21 juillet, 28 juillet et 15 août 1890 .

Sur ce personnage, on consultera avec profit les ouvrages suivants: Elie-JosephArthur Auclair, Le curé Labelle, sa vie et son auvre; ce qu'il était devant ses contemporains, ce $q u$ 'il est devant la postérité, Montréal, Beauchemin, 1930, 271 p. (les papiers de cet auteur. 1866-1946, contenus dans deux boîtes de carton, ont été déposés récemment aux Archives de la ('hancellerie de l'archevêché de Montréal); Arthur Buiss, Au portique des Laurentides, Une paroisse moderne, Le curé Labelle, Québec, Darveau, 1891, 96 p.: renommé pour ses talents d'écrivain,l'auteur trace fidèlement le portrait d'un homme qui s'est voué à l'œuvre de la colonisation. Nous ne pouvons passer sous silence cette étude récente : Hélène Tassé, Le curé Labelle et la région Labelle (1879-1891), thèse présentée au Département d'Histoire de l'Université d'Ottawa en 1968 pour l'obtention de la maîtrise ès arts, xxi-224 p.; malgré une bibliographie qui comporte des sources manuscrites et imprimées fort intéressantes, l'auteur cerne le sujet avec difficultés et incohérence même, à l'occasion. La vie et l'œuvre de ce prêtre, colonisateur et sous-ministre (pour employer la terminologie actuelle), restent à écrire: les sources disponibles attendent le chercheur chevronné.

32 Pour une description des papiers, conservés à Mistassini, cf. notre étude: L'ordre de Cîteaux et son établissement dans la province de Québec..., p. xviii; on pourra également voir dans cette analyse (passim) le rôle qu'a joué le personnage lors de l'établissement des moines à Mistassini.
33 Ibid., p. xxiii.
34 Ibid., p. xvii. 
chercheurs accepteront d'être privés d'une documentation qui est la seule à leur permettre d'expliquer, avec exemples à l'appui, l'influence prépondérante de l'Église sur l'évolution de la société canadiennefrançaise.

L'intérêt qu'on porte aux archives du personnel religieux, susceptibles d'être retrouvées dans les presbytères, s'inscrit donc dans cette perspective. Il va sans dire, toutefois, que les démarches ne s'arrêtent pas au niveau des presbytères, car beaucoup de dépôts d'archives conservent encore des fonds privés du clergé qui demeurent trop peu connus. Une indication, même vague et indéterminée, de leurs préposés sera toujours la bienvenue; elle pourra même être donnée au chercheur lors de la publication de l'inventaire ${ }^{35}$.

Les archives paroissiales, comme on vient de s'en rendre compte, sont d'une richesse trop ignorée; elles devraient pourtant intéresser les jeunes historiens en quête de sujets de recherches dignes de leurs ambitions ${ }^{36}$.

Voilà comment se présente l'inventaire sommaire des sources manuscrites, conservées au Saguenay-Lac-Saint-Jean. Nous sommes en face d'un travail d'envergure qui requiert une méthode précise et beaucoup d'énergies - l'exemple des paroisses le démontre assez bien. Cependant, nous savons qu'une telle entreprise ne restera pas vaine, car des documents nouveaux seront connus des chercheurs. Lorsque plusieurs séries auront été ressassées par des historiens sérieux, nous connaîtrons sûrement mieux notre passé et nous pourrons expliquer davantage les activités et la mentalité de la majeure partie de notre population qui s'est accrochée solidement - du moins jusqu'à ces dernières années - aux traditions de toutes sortes qui caractérisaient les sociétés chrétiennes de l'époque moderne.

André CôtÉ, L.èsL̄., M.A., D.E.N.S., Professeur d'Histoire,

Département des Sciences humaines,

Université du Québec à Chicoutimi.

35 Toute information en ce sens pourra être envoyée à André CôtÉ, professeur d'Histoire, Université du Québec à Chicoutimi, Chicoutimi, Province de Quéhec.

36 Nous sommes heureux de signaler qu'un finissant de notre Universite - qui est en même temps recherchiste dans le projet d'inventaire des archives régionales - s'oriente déjà vers les études paroissiales, au niveau de la maîtrise. Il s'agit en l'occurrence de monsieur Rémi Tremblay; ce dernier analysera les débuts de la paroisse Saint-Jérôme. Monsieur Philippe Sylvain, professeur d'Histoire à l'Université Laval, a accepté de diriger cette recherche. Les excellentes études, relatives au monde rural français de l'Ancien Régime, serviront évidemment de guides à ce jeune chercheur. 